\title{
Evaluation of transport properties of biomembranes by means of Peusner network thermodynamics
}

\author{
KORNELIA M. BATKO ${ }^{1 *}$, ANDRZEJ ŚLĘZAK ${ }^{2}$, WIESŁAW PILIS ${ }^{2}$ \\ ${ }^{1}$ Department of Business Informatics, University of Economics in Katowice, Katowice, Poland. \\ ${ }^{2}$ Department of Health Science, Jan Dlugosz University, Częstochowa, Poland.
}

\begin{abstract}
Purpose: The $R$ version of the Kedem-Katchalsky-Peusner (KKP) network equations is one of the basic research tools for membrane transport. For binary solutions of non-electrolytes containing a solvent and one solute, these equations include the Peusner resistance coefficients. The aim of the study was to assess the transport properties of biomembranes on the basis of the concentration characteristics of the coefficients: resistance, coupling, energy conversion efficiency and degraded and free energy fluxes. Methods: The subject of the study were polymer biomembranes used as a membrane dressing (Bioprocess) and used in hemodialysis (Nephrophan, Ultra-flo) with the coefficients of hydraulic permeability $\left(L_{p}\right)$, reflection $(\sigma)$ and diffusion permeability $(\omega)$ for aqueous glucose solutions. The research method was the $R$ version of the KKP network equations for binary solutions of non-electrolytes. Results: We developed a procedure for evaluation the transport properties of membranes. This procedure requires the calculation of the dependence of the following coefficients: Peusner resistance, Kedem-Caplan-Peusner coupling, Caplan-Peusner energy conversion efficiency, Peusner coupling, and the dissipated energy and free energy fluxes on the mean glucose concentration. Results show that the values of the Peusner resistance coefficients, the Kedem-Caplan-Peusner coupling, the Caplan-Peusner energy conversion efficiency, and the Peusner coupling depend on the mutual relationship between the coefficients $L_{p}, \sigma, \omega$ and $C$. In turn, the value of the dissipated energy and free energy fluxes it is also determined by the values of the volume and diffusion fluxes. Conclusions: The presented procedure for evaluation transport properties of membranes can be helpful in explaining the mechanisms of membrane transport and conducting energy analyzes of membrane processes. Therefore, this procedure can be used for selection of a suitable membrane for practical (eg., industrial or medical) applications.
\end{abstract}

Key words: membrane transport, Kedem-Katchalsky-Peusner equations, polymer biomembrane, transport coefficients of membrane, energy dissipation

\section{Introduction}

Many types of synthetic membranes are used in modern therapy and medical diagnostics and act as a selectively permeable barrier between tissue and environment (e.g., membrane dressings or hemodializers) or between trapped drug and environment (controlled release), etc. [1], [7], [18], [24], [26]. The models of membrane transport developed within Onsager linear non-equilibrium thermodynamics (LNET) and Peusner network thermodynamics (Peusner NT) are convenient tools for studying membrane selectiv- ity [12], [6], [9], [16]. One of the most important is the Kedem-Katchalsky-Peusner model equations [16], [2], [20], [21], [3]. The classic Kedem-Katchalsky model containing transport equations introduces transport coefficients in the character of fitted parameters to the description of membrane transport [8]. For nonelectrolyte solutions, these are the coefficients of: hydraulic permeability $\left(L_{p}\right)$, reflection $(\sigma)$ and solute permeability $(\omega)$.

The fundamental quantity in Onsager thermodynamics is the dissipation function $(\Phi)$, which is described by the expression $\Phi=T\left(d_{i} S / d t\right)$ (where $T$ is the absolute temperature and $d_{i} S / d t$ - the production of

\footnotetext{
* Corresponding author: Kornelia M. Batko, Department of Business Informatics, University of Economics in Katowice, 2B Bogucicka, 40287 Katowice, Poland. E-mail: kornelia.batko@ue.katowice.pl

Received: December 16th, 2020

Accepted for publication: March 24th, 2021
} 
internal entropy) [6], [8]. For isothermal processes, $\Phi=\Sigma_{i} J_{i} X_{i}$. If the forces $\left(X_{i}\right)$ and the fluxes $\left(J_{i}\right)$ are related by an equation of the form $X_{i}=\Sigma_{j} R_{i j} J_{j}$, the matrix of coefficients $R_{i j}(i, j \in\{1,2, \ldots, n\})$ is symmetrical. The degree of coupling $r_{i j}$ results from the relationship between forces and fluxes [9] and for diluted and homogeneous solutions is determined by the relations $r_{i j}=-R_{i j}\left(R_{i i} R_{j j}\right)^{-0.5}$. Besides, the second law of thermodynamics imposes the condition $R_{i i} R_{j j} \geq\left(R_{i j}\right)^{2}$, which means that $r_{i j}$ is limited by the relation $-1 \leq r_{i j} \leq+1$. Considering the $r_{i j}$ factor, Kedem and Caplan presented the expression of the maximum energy conversion efficiency $\left(e_{\max }\right)$ [5]. In turn, Peusner proposed a coupling parameter called "super $Q_{R}$ " [14], [15].

The idea of network thermodynamics (Peusner NT) was presented in 1970 by Leonardo Peusner [13]. $\mathrm{He}$ obtained network forms of $\mathrm{K}-\mathrm{K}$ equations by performing symmetrical and/or hybrid transformation of classical Kedem-Katchalsky equations [15], [16]. For binary solutions of nonelectrolytes (homogeneous and non-homogeneous), there are two symmetrical and two hybrid forms of $\mathrm{K}-\mathrm{K}$ equations. Symmetrical forms of these equations contain Peusner matrix coefficients: $R_{i j}$ and $L_{i j}$ (for homogeneous solutions) and $R_{i j}^{z}$ and $L_{i j}^{z}$ (for non-homogeneous solutions), while hybrid forms include Peusner coefficients: $P_{i j}$ and $H_{i j}$ (for homogeneous solutions) and $P_{i j}^{z}$ and $H_{i j}^{z}$ (for non-homogeneous solutions) $(i, j \in\{1,2\}, \mathrm{z}=\mathrm{A}, \mathrm{B})$ [2], [3], [20], [21]. It should be noted that solutions which are well stirred mechanically are considered homogeneous [9]. In turn, nonhomogeneous solutions are solutions in which concentration polarization occurs, consisting in the formation of concentration boundary layers (CBLs) on both sides of the membrane separating solutions [2]-[4], [20], [21]. These layers serve as additional kinetic barriers for rapidly penetrating substances through membranes in artificial and biological systems [4], [27]. It should be noted that the coefficients $R_{i j}, L_{i j} R_{i j}^{z}$ or $L_{i j}^{z}$, originate directly from Onsager thermodynamics, and the coefficients $P_{i j}, H_{i j}, P_{i j}^{z}$ and $H_{i j}^{z}$ are a consequence of the application of network thermodynamics techniques [2]-[4], [20], [21].

In the present paper, the $R$ form Kedem-Katchalsky-Peusner model equations was used to assess the transport properties of biomembranes. The concentration characteristics of the Peusner $\left(R_{11}, R_{12}, R_{21}, R_{22}\right)$, coupling $\left(r_{12}, r_{21}\right)$, energy conversion efficiency $\left(\left(e_{12}\right)_{r}\right.$, $\left.\left(e_{21}\right)_{r}\right)$ and $Q_{R}$ energy conversion coefficients were calculated on the basis of the transport parameters: hydraulic permeability $\left(L_{p}\right)$, reflection $(\sigma)$ and solute permeability $(\omega)$. The values of parameters $L_{p}, \sigma$ and $\omega$ were determined in a series of independent experiments for aqueous glucose solutions and membranes Bioprocess, Nephrophan and Ultra-flo 145 Dialyzer, according to the procedure described in [9]. In addition, the concentration characteristics of the dissipation function $\left(\Phi_{S}\right)_{R}$ and the concentration characteristics of the free energy production function $\left(\Phi_{F}\right)_{R}$ were also calculated.

\section{Materials and methods}

\subsection{Mathematical model of transport in the membrane cell}

We considered the single-membrane system presented in Fig. 1. In this system, the membrane (M) is located in vertical plane and separates two homogeneous solutions with $C_{r}$ and $C_{l}$ concentrations $\left(C_{r} \geq C_{l}\right)$ and hydrostatic pressures $P_{r}$ and $P_{l}\left(P_{r}>P_{l}, P_{r}=P_{l}\right.$ or $\left.P_{r}<P_{l}\right)$. This membrane treated as a "black box" type is isotropic, symmetrical, electroneutral and selective for solvent and dissolved substance. We decided to consider only isothermal and stationary processes of membrane transport, for which the measure is the volume $\left(J_{v}\right)$ and solute $\left(J_{s}\right)$ fluxes. The membrane transport properties are characterized by the coefficients: hydraulic permeability $\left(L_{p}\right)$, reflection $(\sigma)$ and solute permeability $(\omega)$.

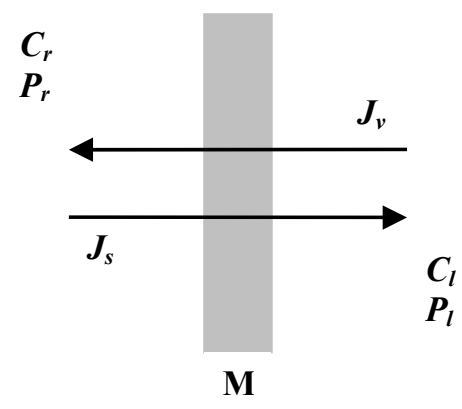

Fig. 1. The single-membrane system: $\mathrm{M}-$ membrane, $J_{v}$ - volume flux, $J_{s}-$ solute flux, $C_{r}$ and $C_{l}-$ concentrations of solute separated by membrane, $P_{r}$ and $P_{l}$-hydrostatic pressures

The volume $\left(J_{v}\right)$ and solute $\left(J_{s}\right)$ fluxes can be expressed using the classic Kedem-Katchalsky equations [9]

$$
\begin{gathered}
J_{v}=L_{p} \Delta P-L_{p} \sigma \Delta \pi \\
J_{s}=\omega \Delta \pi+\bar{C}(1-\sigma) J_{v},
\end{gathered}
$$


where: $J_{v}$ - volume flux, $J_{s}$ - solute flux, $\Delta P=P_{r}-P_{l}$ - difference of hydrostatic pressure, $\Delta \pi=R T\left(C_{r}-C_{l}\right)$ - difference of osmotic pressure, $R T$ - product of gas constant and absolute temperature, $L_{p}$ - hydraulic permeability coefficient, $\sigma$ - reflection coefficient, $\omega$ - solute permeability coefficient, $\bar{C}=$ $\left(C_{r}-C_{l}\right)\left[\ln \left(C_{r} / C_{l}\right)\right]^{-1}$ - average solute concentration.

Relatively simple algebraic transformations allow to write Eqs. (1) and (2) in the following form

$$
\begin{gathered}
\Delta P-\Delta \pi=\frac{\omega+L_{p} \bar{C}(1-\sigma)^{2}}{L_{p} \omega} J_{v}-\frac{1-\sigma}{\omega} J_{s} \\
\frac{\Delta \pi}{\bar{C}}=-\frac{1-\sigma}{\omega} J_{v}+\frac{1}{\bar{C} \omega} J_{s} .
\end{gathered}
$$

The above equations are called transformed Kedem-Katchalsky equations or the $R$ version of Kedem-Katchalsky-Peusner equations [4], [14], [15], [21].

Equations (3) and (4) presented in matrix form contain Peusner coefficients $R_{i j}(i, j \in\{1,2\})$ and can be written in the form

$$
\begin{gathered}
{\left[\begin{array}{c}
\Delta P-\Delta \pi \\
\frac{\Delta \pi}{\bar{C}}
\end{array}\right]=[R]\left[\begin{array}{l}
J_{v} \\
J_{s}
\end{array}\right],} \\
{[R]=\left[\begin{array}{ll}
R_{11} & R_{12} \\
R_{21} & R_{22}
\end{array}\right]=\left[\begin{array}{cc}
\frac{\omega+L_{p} \bar{C}(1-\sigma)^{2}}{L_{p} \omega} & -\frac{1-\sigma}{\omega} \\
-\frac{1-\sigma}{\omega} & \frac{1}{\bar{C} \omega}
\end{array}\right] .}
\end{gathered}
$$

In addition, the determinant of the matrix $[R]$ is given by the equation

$$
\operatorname{det}[R]=\frac{1}{L_{p} \bar{C} \omega} \equiv R_{\text {det }} .
$$

The coefficients $R_{i j}(i, j \in\{1,2\})$ and $R_{\text {det }}$ are the $R$ form of the Peusner coefficients for membrane transport of binary solutions. The coefficients $R_{i j}$ can be used in the calculation of Kedem-Caplan-Peusner coupling coefficients $r_{12}$ and $r_{21}$, Peusner coupling "super $Q_{R}$ " parameter and Kedem-Caplan-Peusner [16] coefficient of maximum energy conversion efficiency $\left(e_{\max }\right)_{r}$.

\subsection{Mathematical model of energy conversion in the membrane cell}

In a system containing a membrane separating solutions of different concentrations, internal energy $(U)$ is converted into free energy, which can be converted into useful work and/or into other types of energy $(F)$ and associated energy (TS, $T$ - absolute temperature, $S$ - entropy) [6], [9]. The rates of change of these variables are related to each other by the equation

$$
\frac{d U}{d t}=\frac{d F}{d t}+T \frac{d S}{d t}=\frac{d F}{d t}+T\left(\frac{d_{i} S}{d t}+\frac{d_{e} S}{d t}\right)
$$

where $d_{i} S / d t$ - means the rate at which entropy is created in the membrane system by irreversible processes (accumulated entropy flow), while $d_{e} S / d t$ - the rate of entropy exchange with an environment (entropy flow to the environment). Assuming that $d_{e} S / d t=0$ we get $d s / d t$ $=d_{i} S / d t$. Dividing both sides of this equation by the area A, we get the Eq. (8) in the flux version [6], [9]

$$
\Phi_{U}=\Phi_{F}+\Phi_{S},
$$

where $\Phi_{U}=A^{-1} d U / d t$ - flux of internal energy, $\Phi_{F}=$ $A^{-1} d F / d t$ - flux of free energy, $\Phi_{S}=A^{-1} d_{i} S / d t$ bound energy flux (function of energy dissipation per unit area). $\Phi_{S}$ can be calculated using the equation [9], [23], [25]

$$
\Phi_{s}=J_{v}(\Delta P-\Delta \pi)+J_{s} \frac{\Delta \pi}{\bar{C}} .
$$

After considering Eqs. (3) and (4) in Eq. (10), we obtain the $R$ version of the dissipation function $\left(\Phi_{S}\right)_{R}$ for a single-membrane system

$$
\left(\Phi_{S}\right)_{R}=R_{11} J_{v}^{2}+\left(R_{12}+R_{21}\right) J_{v} J_{s}+R_{22} J_{s}^{2} .
$$

In this expression, $\left(\Phi_{S}\right)_{R}$ is the $R$ form of the flux of dissipated energy, i.e., the temporal change in energy per unit of membrane surface expressed in $\mathrm{J} / \mathrm{m}^{2} \mathrm{~s}$ or W/m $\mathrm{m}^{2}$. We can calculate the free energy flux, $\left(\Phi_{F}\right)_{R}$, using the expression below

$$
\left(e_{\max }\right)_{r}=\frac{\left(\Phi_{F}\right)_{R}}{\left(\Phi_{U}\right)_{R}}=\frac{\left(\Phi_{F}\right)_{R}}{\left(\Phi_{F}\right)_{R}+\left(\Phi_{S}\right)_{R}},
$$

where $\left(e_{\max }\right)_{r}$ is the coefficient of maximum energy conversion efficiency. As suggested by Kedem and Caplan [10] and Peusner [16], the coefficient $\left(e_{\max }\right)_{r}$ can be represented by the expression

$$
\begin{gathered}
\left(e_{21}\right)_{r}=\frac{R_{21}^{2}}{R_{11} R_{22}\left(1+\sqrt{1-\frac{R_{12} R_{21}}{R_{11} R_{22}}}\right)^{2}}=\left(e_{12}\right)_{r} . \\
=\frac{R_{12}^{2}}{R_{11} R_{22}\left(1+\sqrt{1-\frac{R_{12} R_{21}}{R_{11} R_{22}}}\right)^{2}}=\left(e_{\max }\right)_{r} .
\end{gathered}
$$


The values of the coefficients $\left(e_{\max }\right)_{r}$ are limited by the relation $0 \leq\left(e_{\max }\right)_{r} \leq+1$. Transforming Eq. (12) we can get

$$
\left(\Phi_{F}\right)_{R}=\frac{\left(e_{\max }\right)_{r}}{1-\left(e_{\max }\right)_{r}}\left(\Phi_{S}\right)_{R} .
$$

From the above equation, we can calculate the amount of free energy in that system.

\subsection{Method for measuring the volume and solute fluxes and transport parameters}

Measurement system for evaluation of membrane transport, was described in the previous paper [22]. It consisted of two Plexiglas vessels $(r, l)$ with a capacity of $V_{\mathrm{c}}=200 \mathrm{~cm}^{3}$, separated by a membrane (M) with an area of $3.36 \mathrm{~cm}^{2}$. The vessel $r$ was filled with aqueous glucose solution with concentration $C_{r}$, and the vessel $l$ with concentration $C_{l}$. A calibrated pipette was attached to vessel $r$ to measure the volume increase $(\Delta V)$ in this vessel caused by volume flow through membrane. The vessel $l$ was equipped with a solution reservoir $(\mathrm{N})$ with a concentration $C_{l}$, the position of which could be moved vertically, relatively to the pipette along the millimetre scale. The solutions were stirred using mechanical stirrers installed in vessels $r$ and $l$. Volume $\left(J_{v}\right)$ and solute $\left(J_{s}\right)$ fluxes were calculated on the basis of formulas $J_{v}=$ $\Delta V / A \cdot \Delta t$ and $J_{s}=d C \cdot V_{c} / A \cdot \Delta t$, where $A$ - total membrane surface area, $\Delta V$ - increase of volume during time $\Delta t, d C$ - the change of concentration in measurement vessel, $V_{c}$ - volume of measurement vessel. The change of concentration $(d C)$ was determined using optical method [8], for isothermal conditions $(T=295 \mathrm{~K})$ for membranes: Bioprocess (index B),
Nephrophan (index $N$ ) and Ultra-flo 145 Dialyzer (index $U$ ). The transport properties of these biomembranes were determined by the: hydraulic permeability $\left(L_{p}\right)$, diffusion permeability $(\omega)$ and reflection $(\sigma)$ coefficients. These coefficients were calculated based on definitions that result from Eqs. (1) and (2): $L_{p}=$

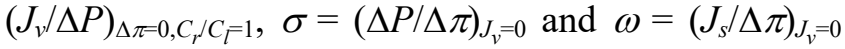
[8]. Each experiment was repeated three times. The results summarized in Table 1 and in Fig. 3.

\subsection{Biomembranes characteristics}

In Figure 2, images of Bioprocess, Nephrophan and Ultra-flo 145 Dialyzer membranes obtained with a scanning electron microscope (Zeiss Supra 35) are shown. The image shown in Fig. 2a shows the microfiber structure of the Bioprocess membrane, Fig. 2b shows the image of the solid structure of the Nephrophan membrane and in Fig. 2c shows the microfiber structure of the Ultra-flo 145 Dialyzer membrane.

Bacterial cellulose membranes Bioprocess $^{\circledR}$, Biofill, Productos Biotechnologicos, SA, Curitiba, Brasile) are produced in flat sheets and their membrane structure is formed by microcellulose fibers produced by Acetobacter Xylinum. In Figure 2a, it can be seen that the membrane structure is in the form of a network of braided microfibrils consisting of cellulose fibers with a cross-section of $0.1-0.2 \mu \mathrm{m}$ and a length of several micrometers. Such a network of braided fibers gives the membrane high elasticity and tear resistance. The Bioprocess membrane has a layered structure, with each layer consisting of braided cellulose fibers [8], [19].

Nephrophan $^{\circledR}$ (Orwo VEB Filmfabrik, Wolfen, Germany) biomembrane, is a microporous, highly hydrophylic and electroneutral membrane made of regenerated cellulose [11]. The structure of this membrane is compact. This membrane is used in urology (a)

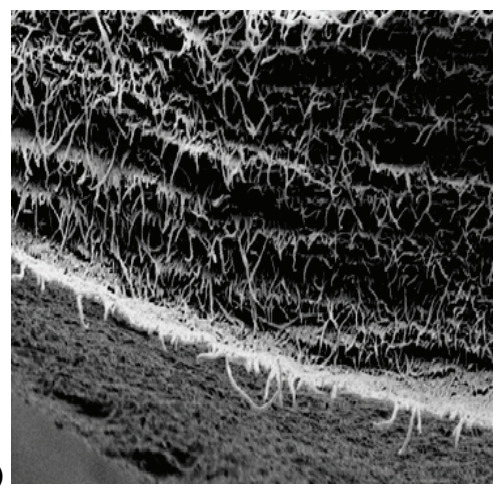

(b)

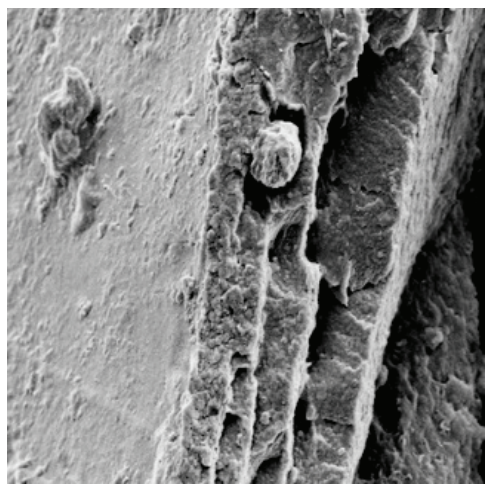

(c)

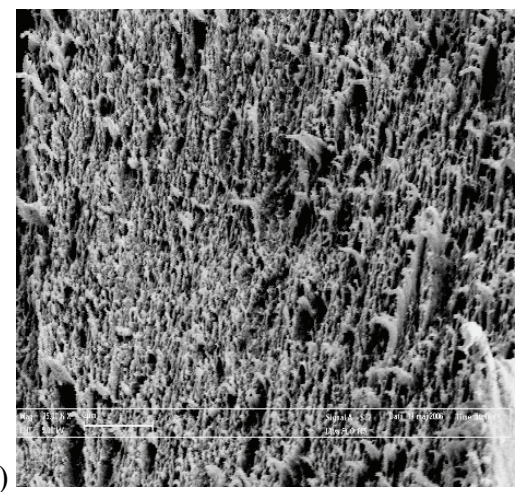

Fig. 2. Images of membranes surfaces obtained from scanning electron microscope: (a) cross-section of the Bioprocess membrane (magnification 10000 times) [8], (b) surface and cross-section of the Nephrophane membrane (magnification 15000 times) [22], (c) cross-section of the Ultra-flo 145 Dialyser membrane (magnification 10500 times) 
in ganglion hemodialysers, due to their high-pressure strength, and in ophthalmology and laryngology - for controlled release of drugs [17].

Ultra-flo 145 Dialyzer $^{\circledR}$ (Artificial Organs Division, Travenol Laboratories, Brussels, Belgium) (with regenerated cellulose membrane) is a ultrafiltration, microporous, hydrophylic and electroneutral biomembrane used in urology [18].

\section{Results}

\subsection{Determination of membrane transport parameters}

The values of $L_{p}, \omega$ and $\sigma$ coefficients for biomembranes Bioprocess (index B), Nephrophan (index $N$ ) and Ultra-flo 145 Dialyzer (index $U$ ) and aqueous glucose solutions, determined in a series of independent experiments carried out according to the procedure described in paper [8], are summarized in Table 1.

Table 1. Values of biomembrane transport parameters for glucose

\begin{tabular}{|l|c|c|c|}
\hline \multirow{2}{*}{$\begin{array}{c}\text { Membrane transport } \\
\text { parameters }\end{array}$} & \multicolumn{3}{|c|}{ Membrane } \\
\cline { 2 - 4 } & Bioprocess & Nephrophan & Ultra-flo 145 \\
\hline$L_{p}\left[\mathrm{~m}^{3} \mathrm{~N}^{-1} \mathrm{~s}^{-1}\right]\left(\times 10^{12}\right)$ & $36.2 \pm 2.1$ & $5.0 \pm 0.1$ & $1.3 \pm 0.1$ \\
\hline$\sigma\left(\times 10^{2}\right)$ & $0.84 \pm 0.02$ & $6.8 \pm 0.2$ & $15 \pm 1.2$ \\
\hline$\omega\left[\mathrm{molN}^{-1} \mathrm{~s}^{-1}\right]\left(\times 10^{10}\right)$ & $64.1 \pm 3.1$ & $8.0 \pm 0.5$ & $4.0 \pm 0.1$ \\
\hline
\end{tabular}

It was found that the values of $L_{p}, \sigma$ and $\omega$ for these membranes are independent of $(\bar{C})$. For tested membranes, the dependencies can be written as $L_{p B} \gg$ $L_{p N}>L_{p U}, \sigma_{B}<\sigma_{N}<\sigma_{U}$ and $\omega_{B}<\omega_{N}>\omega_{U}$ when comparing the values of the $L_{p}$ and $\omega$ coefficients. The transport parameters listed in Table 1 were used to calculate the Peusner coefficients $R_{11}=f(\bar{C})$ (Fig. 4a), $R_{12}=R_{21}=f(\bar{C})$ (Fig. 4b), $R_{22}=f(\bar{C})$ (Fig. 4c) and $R_{\text {det }}=f(\bar{C})$ (Fig. 4d).

\subsection{Concentration dependencies of $J_{v}$ and $J_{s}$}

The dependencies $J_{v}=f(\bar{C})$ and $J_{s}=f(\bar{C})$ are shown in Figs. 3a and 3b for Bioprocess (B), Nephrophan (N) and Ultra-flo 145 Dialyzer membranes (U). The relation $J_{v}=f(\bar{C})$ presented in Fig. 3a shows that for the same values of $(\bar{C})$ the relation $J_{v N}>J_{v B}>J_{v U}$ is satisfied. While the relation $J_{s}=f(\bar{C})$ presented in Fig. 3b shows that for the same values of $(\bar{C})$ the relation $J_{S s B}>J_{S N}>J_{S U}$ is satisfied.

\subsection{Concentration dependence of coefficients $\boldsymbol{R}_{i j}$ and $\boldsymbol{R}_{d e t}$}

The values $R_{11}, R_{12}=R_{21}, R_{22}$ and $R_{\text {det }}$ were calculated using Eqs. (6) and (7). In Figure 4a, shows the linear dependence of $R_{11}=f \bar{C}$ for Bioprocess (graphs 1), Nephrophan (graphs 2) and Ultra-flo 145 Dialyzer (graphs 3) biomembranes is shown. In the tested range of $\bar{C}$, the value of the coefficient $\left(R_{11}\right)_{B}$ increases linearly from $2.77 \times 10^{10} \mathrm{Ns} \cdot \mathrm{m}^{-3}$ to 3.09
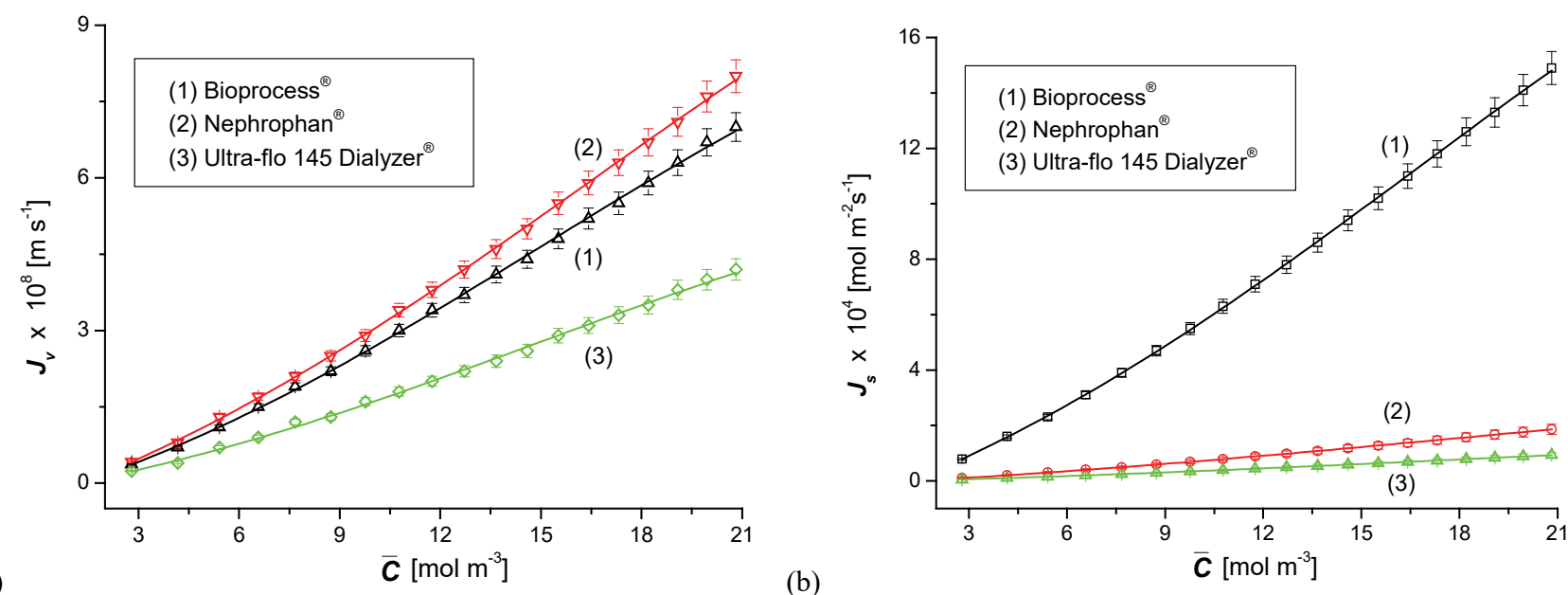

Fig. 3. Graphic illustration of dependencies $J_{v}=f(\bar{C})$ (a) and $J_{s}=f(\bar{C})$ (b) for membranes Bioprocess, Nephrophan and Ultra-flo 145 Dialyzer for aqueous glucose solutions 
$\times 10^{10} \mathrm{Ns} \cdot \mathrm{m}^{-3}$, of the coefficient $\left(R_{11}\right)_{N}$ increases linearly from $20.07 \times 10^{10} \mathrm{Ns} \cdot \mathrm{m}^{-3}$ to $22.34 \times 10^{10} \mathrm{Ns} \cdot \mathrm{m}^{-3}$ and of the coefficient $\left(R_{11}\right)_{U}$ increases linearly from 77.02 $\times 10^{10} \mathrm{Ns} \cdot \mathrm{m}^{-3}$ to $80.82 \times 10^{10} \mathrm{Ns} \cdot \mathrm{m}^{-3}$. This means that for the same values of $\bar{C}$ the relation $\left(R_{11}\right)_{B}<\left(R_{11}\right)_{N}$ $<\left(R_{11}\right)_{U}$ is satisfied. From the above, it follows that the coefficient $R_{11}$ act as the hydraulic resistance coefficient.

From Figure $4 \mathrm{~b}$ it follows that $R_{12}=R_{21}$ are independent of $\bar{C}$ and negative. For the tested membranes, the values of these coefficients are: $\left(R_{12}=R_{21}\right)_{B}=-1.55$ $\times 10^{8} \mathrm{Ns} \cdot \mathrm{mol}^{-1},\left(R_{12}=R_{21}\right)_{N}=-0.12 \times 10^{10} \cdot \mathrm{Ns} \mathrm{mol}^{-1}$ and $\left(R_{12}=R_{21}\right)_{U}=-0.21 \times 10^{10} \mathrm{Ns} \cdot \mathrm{mol}^{-1}$. Moreover, for the same values of $\bar{C}$ the relation $\left(R_{12}=R_{21}\right)_{B}>\left(R_{12}=R_{21}\right)_{N}$ $>\left(R_{12}=R_{21}\right)_{U}$ is satisfied. It follows that the coefficients $R_{12}$ and $R_{21}$ act as the diffusion resistance coefficient.

Figure $4 \mathrm{c}$ shows that the curves 1 (for Bioprocess), 2 (for Nephrophan) and 3 (for Ultra-flo 145 Dialyzer) illustrating the dependencies $R_{22}=f \bar{C}$ are hyperbolas. In the examined range of $\bar{C}$, the value of the coeffi- cients: $\left(R_{22}\right)_{B}$ decrease nonlinearly from $2.25 \times 10^{8}$ $\mathrm{m}^{3} \mathrm{Ns} \cdot \mathrm{mol}^{-2}$ to $0.07 \times 10^{8} \mathrm{~m}^{3} \mathrm{Ns} \cdot \mathrm{mol}^{-2},\left(R_{22}\right)_{N}$ increases linearly from $18.0 \times 10^{8} \mathrm{~m}^{3} \mathrm{Ns} \cdot \mathrm{mol}^{-2}$ to $0.6 \times 10^{8}$ $\mathrm{m}^{3} \mathrm{Ns} \cdot \mathrm{mol}^{-2},\left(R_{22}\right)_{U}$ decrease nonlinearly from 36.01 $\times 10^{8} \mathrm{~m}^{3} \mathrm{Ns} \cdot \mathrm{mol}^{-2}$ to $1.15 \times 10^{8} \mathrm{~m}^{3} \mathrm{Ns} \cdot \mathrm{mol}^{-2}$.

This means that for the same values of $\bar{C}$ the relation $\left(R_{22}\right)_{B}<\left(R_{22}\right)_{N}<\left(R_{22}\right)_{U}$ is satisfied. Similarly, it is shown in Fig. $4 d$ that the curves 1 (for Bioprocess), 2 (for Nephrophan) and 3 (for Ultra-flo 145 Dialyzer) illustrating the dependence $R_{d e t}=f \bar{C}$ are hyperbolas. In the tested range of $\bar{C}$, the value of the coefficient $\left(R_{d e t}\right)_{B}$ decreased nonlinearly from $0.062 \times$ $10^{20} \mathrm{~N}^{2} \mathrm{~s}^{2} \cdot \mathrm{mol}^{-2}$ to $0.002 \times 10^{20} \mathrm{~N}^{2} \mathrm{~s}^{2} \cdot \mathrm{mol}^{-2}$, of the coefficient $\left(R_{\text {det }}\right)_{N}$ decrease nonlinearly from $3.61 \times$ $10^{20} \mathrm{~N}^{2} \mathrm{~s}^{2} \cdot \mathrm{mol}^{-2}$ to $0.12 \times 10^{20} \mathrm{~N}^{2} \mathrm{~s}^{2} \cdot \mathrm{mol}^{-2}$ and of the coefficient $\left(R_{22}\right)_{U}$ increases linearly from $27.7 \times$ $10^{20} \mathrm{~N}^{2} \mathrm{~s}^{2} \cdot \mathrm{mol}^{-2}$ to $0.89 \times 10^{20} \mathrm{~N}^{2} \mathrm{~s}^{2} \cdot \mathrm{mol}^{-2}$. The course of these curves shows that for the same values of $\bar{C}$ the relation $\left(R_{d e t}\right)_{B}<\left(R_{d e t}\right)_{N}<\left(R_{d e t}\right)_{U}$ is satisfied.
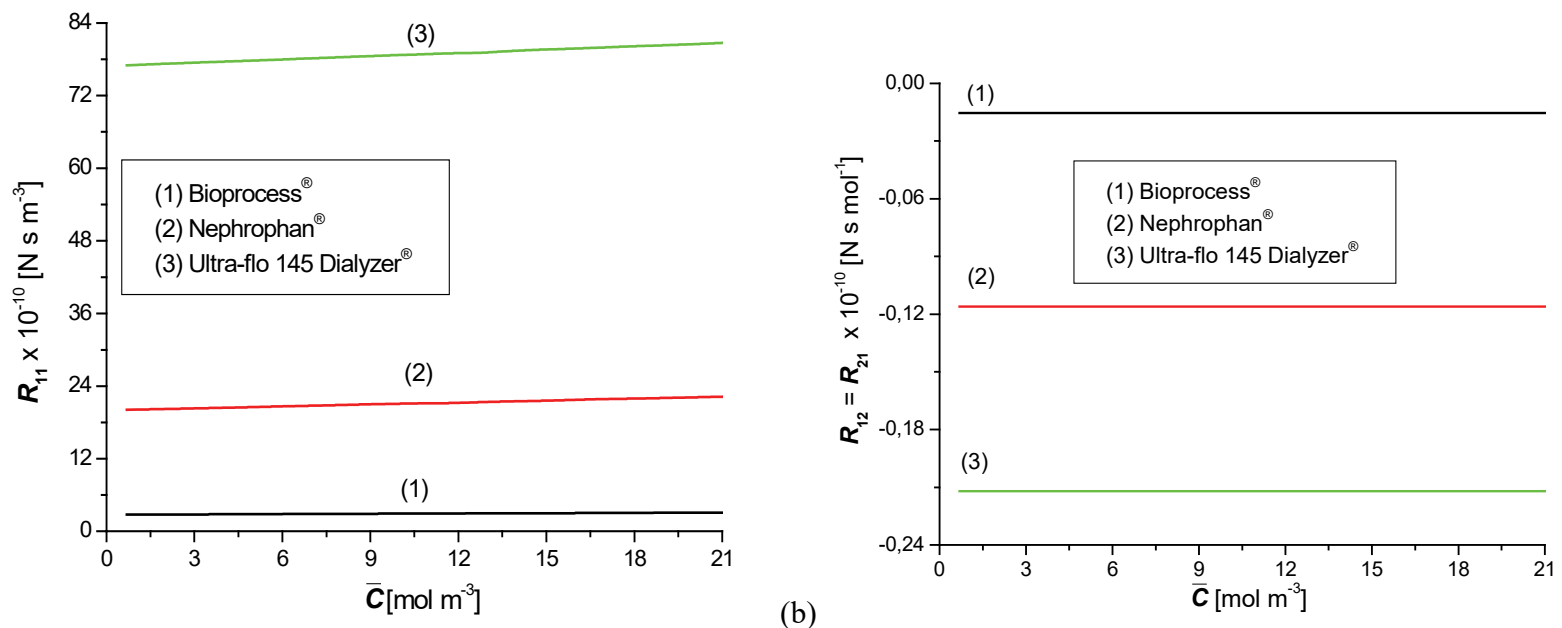

(a)

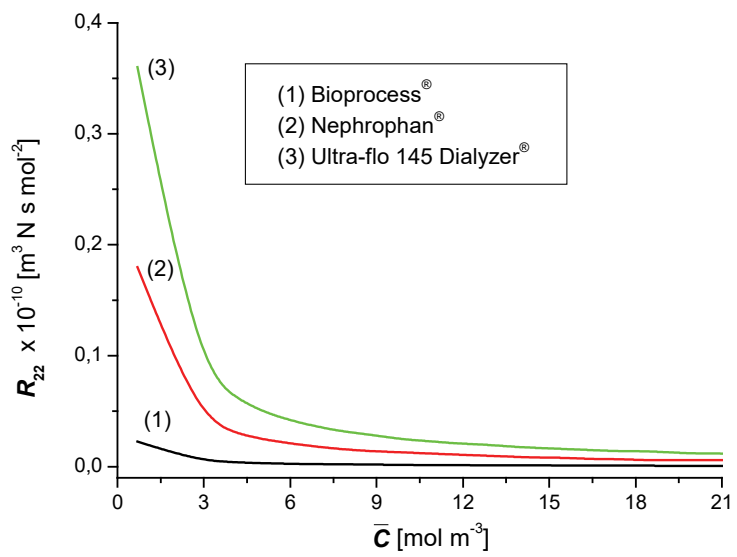

(b)

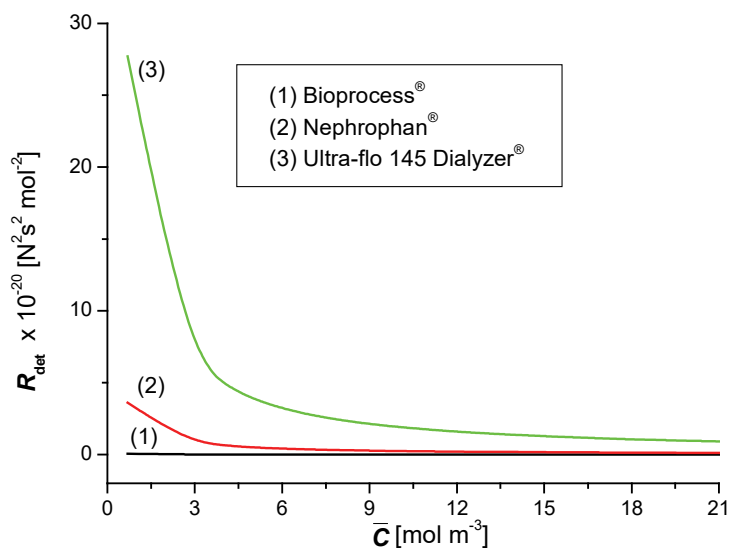

(d)

Fig. 4. Graphic illustration of dependences: $R_{11}=f(\bar{C})(\mathrm{a}), R_{12}=R_{21}=f(\bar{C})(\mathrm{b}), R_{22}=f(\bar{C})(\mathrm{c})$ and $R_{\text {det }}=f(\bar{C})(\mathrm{d})$ suitably for Bioprocess (graphs 1), Nephrophan (graphs 2) and Ultra-flo 145 Dialyzer (graphs 3 ) biomembranes for aqueous glucose solutions 


\section{Discussion}

\subsection{Concentration dependence of $r_{i j}, Q_{R}$ and $\left(e_{i j}\right)_{r}$ coefficients}

Using the $R_{i j}(i, j \in\{1,2\}$ coefficients, it is possible to present the expression for the $r_{12}$ and $r_{21}$ coefficients, using the definition proposed by Kedem and Caplan [10] and Peusner [16]. This expression takes the form

$$
r_{12}=r_{21}=-\frac{R_{12}}{\sqrt{R_{11} R_{22}}}=-\frac{R_{21}}{\sqrt{R_{11} R_{22}}} .
$$

The value of the coefficients $r_{12}=r_{21}=r$ is limited by the relation $-1 \leq r \leq+1$. When $r= \pm 1$, the system is fully coupled, the processes become a single process. When $r=0$, the two processes are completely unconjugated, and no energy conversion interactions occur.

Peusner proposed a $Q_{R}$ coupling parameter called "super $Q_{R}$ " [16]

$$
Q_{R}=\frac{2\left|R_{12} R_{21}\right|}{4 R_{11} R_{22}-2 R_{12} R_{21}}=\frac{r_{12} r_{21}}{2-r_{12} r_{21}} .
$$

The values of the coefficients $Q_{R}$ is limited by the relation $0 \leq Q_{R} \leq+1$. In turn, using Eq. (15), Eq. (13) can be written as

$$
\begin{gathered}
\left(e_{21}\right)_{r}=\frac{r_{21}^{2}}{\left(1+\sqrt{1-r_{12} r_{21}}\right)^{2}} \\
=\left(e_{12}\right)_{r}=\frac{r_{12}^{2}}{\left(1+\sqrt{1-r_{12} r_{21}}\right)^{2}}=\left(e_{\max }\right)_{r} .
\end{gathered}
$$

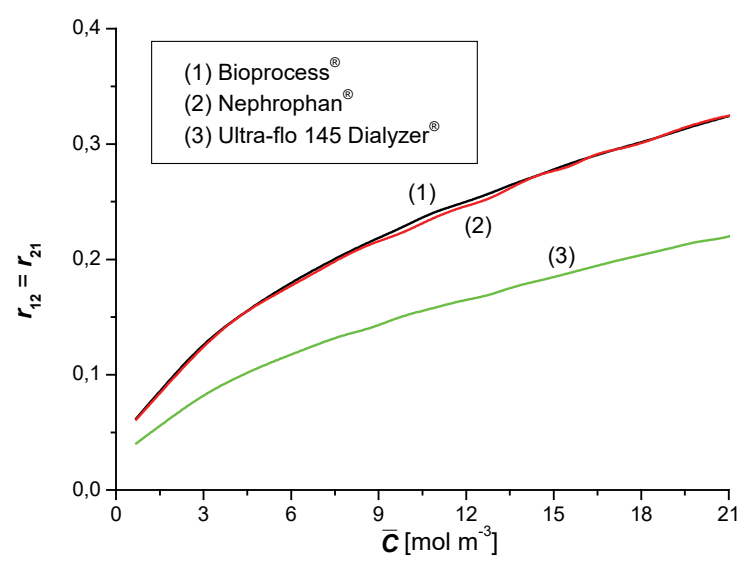

Fig. 5. Graphic illustration of dependencies: $r_{12}=r_{21}=f(\bar{C})$ (a) and $Q_{R}=f(\bar{C})$ (b) suitably for membranes: Bioprocess (graphs 1), Nephrophan (graphs 2) and Ultra-flo 145 Dialyzer (graphs 3) for aqueous glucose solutions (b)

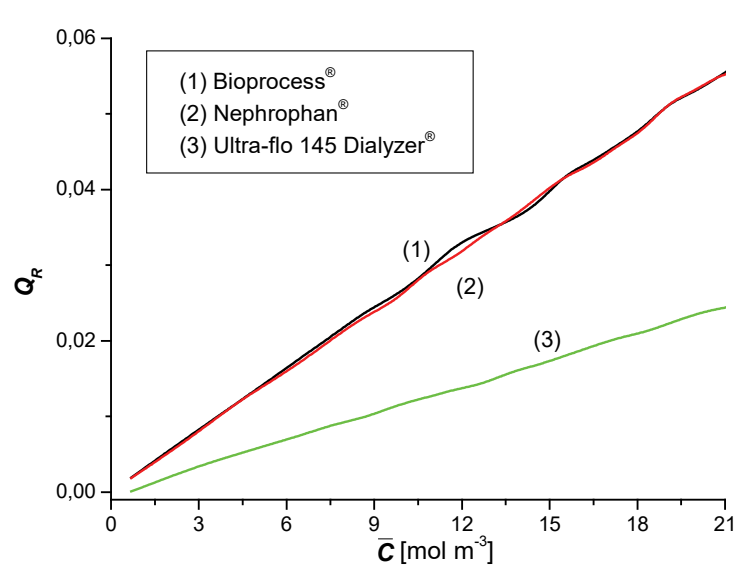

Taking the calculation results presented in Fig. 4a, $4 \mathrm{~b}$ and $4 \mathrm{c}$ in Eqs. (15), (16) and (17), into account, it is possible to calculate Kedem-Caplan-Peusner coupling coefficients $r_{12}$ and $r_{21}$, Peusner coupling "super $Q_{R}$ " parameter and Kedem-Caplan-Peusner [16] coefficients of energy conversion efficiency $\left(e_{12}\right)_{r}$ and $\left(e_{21}\right)_{r}$.

The dependencies $r_{12}=f(\bar{C}), Q_{R}=f(\bar{C})$ and $\left(e_{12}\right)_{r}$ $=f(\bar{C})$ for membranes Bioprocess $(\mathrm{B})$, Nephrophan (N) and Ultra-flo 145 Dialyzer (U). These dependencies are presented in Figs. 5 and 6. In Figure 4a, it is shown that, together with the curves illustrating the dependencies $r_{12}=f(\bar{C})$, we can observe saturated curves. In Fig. 4a, it is shown that for the range $0.7 \mathrm{~mol}$ $\mathrm{m}^{-3} \leq \bar{C} \leq 20.8 \mathrm{~mol} \mathrm{~m}^{-3}$ and Bioprocess membrane, the Kedem-Caplan-Peusner equation is as follows: $0.06 \leq\left(r_{12}\right)_{B} \leq 0.33$. For Nephrophan and Ultra-flo 145 Dialyzer membranes, this equation can be written as $0.06 \leq\left(r_{12}\right)_{N} \leq 0.32$, respectively. Thus, for the studied membranes, the transport processes are partially coupled. This coupling increases with the increase of $\bar{C}$, and causes energy conversion interactions.

When the value of $\bar{C}$ increases, the values of the coefficients $\left(r_{12}\right)_{B}=\left(r_{12}\right)_{N}$ for the Bioprocess, Nephrophan membranes and $\left(r_{12}\right)_{U}$ for Ultra-flo 145 Dialyzer membranes tend asymptotically towards the fixed value. For the investigated concentration range, the conditions $\left(r_{12}\right)_{B} \approx\left(r_{12}\right)_{N} \rightarrow 0.35$ and $\left(r_{12}\right)_{U} \rightarrow 0.25$ are fulfilled. This means that the solvent and solute transport processes are coupled in varying degrees. The strongest coupling occurs for the Bioprocess and Nephrophan membranes, and the weakest - for the Ultra-flo 145 Dialyzer. Using the coefficients $r_{12}$ and $r_{21}$, we can calculate the coefficients $Q_{R},\left(e_{12}\right)_{r}$ and $\left(e_{21}\right)_{r}$. 
The first is a measure of the degree of coupling and the second is the energy conversion efficiency.

In Figure 5b, it is shown that, with the increase of value $\bar{C}$, the value of $Q_{R}$ linearly increases. In Figure $5 \mathrm{~b}$, it is shown that for average concentrations of glucose solutions $(\bar{C})$ in the range $0.7 \mathrm{~mol} \cdot \mathrm{m}^{-3} \leq \bar{C}$ $\leq 20.8 \mathrm{~mol} \cdot \mathrm{m}^{-3}$ for the Bioprocess membrane, the Peusner equation is as follows $0.002 \leq\left(Q_{R}\right)_{B} \leq 0.06$. For Nephrophan and Ultra-flo 145 Dialyzer membranes, these equations can be written as $0.002 \leq$ $\left(Q_{R}\right)_{N} \leq 0.05$ and $0.0001 \leq\left(Q_{R}\right)_{U} \leq 0.025$, respectively. For the same values of $\bar{C}$, the following condition $\left(Q_{R}\right)_{B}=\left(Q_{R}\right)_{N}>\left(Q_{R}\right)_{U}$ is satisfied.

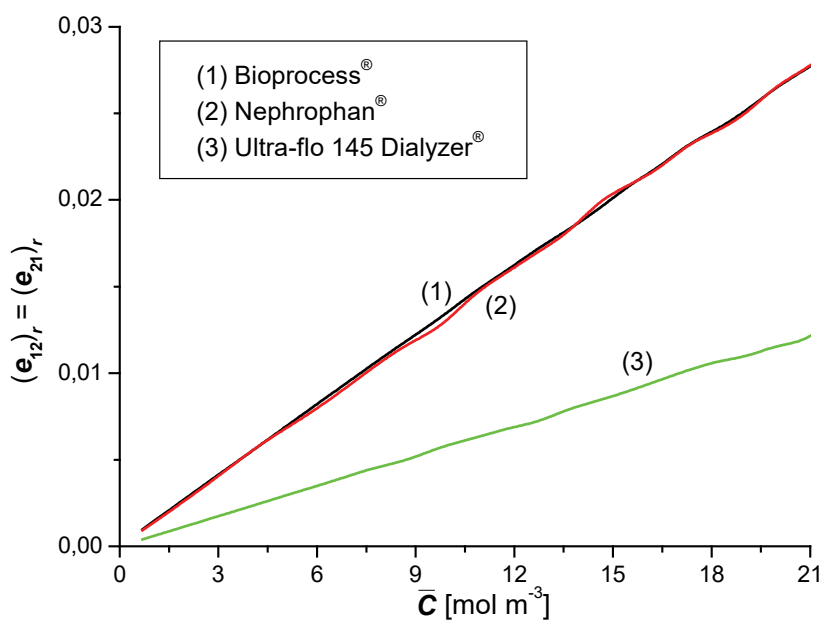

Fig. 6. Graphic illustration of dependencies: $\left(e_{12}\right)_{r}=\left(e_{21}\right)_{r}=f(\bar{C})$

for membranes: Bioprocess (graph 1), Nephrophan (graph 2) and Ultra-flo 145 Dialyzer (graph 3) for aqueous glucose solutions

In Figures $5 \mathrm{a}, 5 \mathrm{~b}$ and 6 , it is shown that for Bioprocess and Nephrophan membranes, although $L_{p B}>$ $L_{p N}, \sigma_{B}<\sigma_{N}, \omega_{B}>\omega_{N}$ for the same values of $\bar{C}$, the relations $\left(r_{12}\right)_{B}=\left(r_{12}\right)_{N},\left(Q_{R}\right)_{B}=\left(Q_{R}\right)_{N}$ and $\left(e_{12}\right)_{r B}=$ $\left(e_{12}\right)_{r N}$. Taking Eqs. (8), (9) or (11) into account, it can take the following form:

$$
L_{p B}\left(1-\sigma_{B}\right)^{2} \omega_{N}=L_{p N}\left(1-\sigma_{N}\right)^{2} \omega_{B} .
$$

From Figure 6, it can be seen that that with the increase of value $\bar{C}$, the values of the coefficient $\left(e_{12}\right)_{r}$ and $\left(e_{21}\right)_{r}$ linearly increase. In Figure 6 , it is shown that for the range $0.7 \mathrm{~mol} \cdot \mathrm{m}^{-3} \leq \bar{C} \leq 20.8 \mathrm{~mol} \cdot \mathrm{m}^{-3}$ for the Bioprocess membrane, the Peusner equation is as follows $0.001 \leq\left(e_{12}\right)_{r B} \leq 0.028$. While, for Nephrophan and Ultra-flo 145 Dialyzer membranes, these equations can be written as $001 \leq\left(e_{12}\right)_{r N} \leq 0.027$ and $0.0004 \leq\left(e_{12}\right)_{r U} \leq 0.012$, respectively. All tested membranes, (due to the low value of the coefficient $\left.\left(e_{12}\right)_{r}\right)$ are characterized by low energy conversion efficiency. For the same values of $\bar{C}$, the condition $\left(e_{12}\right)_{r B}=\left(e_{12}\right)_{r N}>\left(e_{12}\right)_{r U}$ is fulfilled. The results presented in Fig. 6 show that the Bioprocess and Nephrophan membranes are the most effective energy converters.

Using Eq. (6), Eqs. (15)-(17) can be written in following forms

$$
\begin{gathered}
r_{12}=r_{21}=\sqrt{\frac{L_{p}(1-\sigma)^{2} \bar{C}}{\omega+L_{p}(1-\sigma)^{2} \bar{C}}} \\
Q_{R}=\frac{(1-\sigma)^{2} \bar{C}}{\frac{2 \omega}{L_{p}}(1-\sigma)^{2} \bar{C}}, \\
=\left(e_{12}\right)_{r}=\frac{\left[\omega+L_{p}(1-\sigma)^{2} \bar{C}\right]\left(1+\sqrt{\frac{L_{p}(1-\sigma)^{2} \bar{C}}{\omega+L_{p}(1-\sigma)^{2} \bar{C}}}\right)^{2}}{\left(1+Q_{R}\right)\left(1+\sqrt{\frac{1-Q_{R}}{1+Q_{R}}}\right)^{2}} .
\end{gathered}
$$

The above equations allow us to calculate the coefficients $r_{12}, r_{21}, Q_{R},\left(e_{21}\right)_{r}$ and $\left(e_{12}\right)_{r}$ based on the experimentally determined parameters of $L_{p}, \sigma$ and $\omega$.

\subsection{Concentration dependence of fluxes $\left(\Phi_{S}\right)_{R}$ and $\left(\Phi_{F}\right)_{R}$}

In order to calculate the dependence $\left(\Phi_{S}\right)_{R}=f(\bar{C})$ let us consider the Eq. (11) of the dependence $R_{11}=$ $f(\bar{C}), R_{12}=R_{21}=f(\bar{C}), R_{22}=f(\bar{C})$ shown in Figs. $3 \mathrm{a}-3 \mathrm{c}$ and the dependencies $J_{v}=f(\bar{C})$ and $J_{s}=f(\bar{C})$ shown in Figs. 3a and 3b for Bioprocess (B), Nephrophan (N) and Ultra-flo 145 Dialyzer membranes (U). Taking the results of investigations presented in Figs. $4 a-4 c, 3 a$ and $3 b$ in Eq. (11) into account, the following dependencies were obtained $\left(\Phi_{S}\right)_{R}=f(\bar{C})$ and presented in Fig. 7a. This figure shows that for the same values of $\bar{C}$ the relation $\left(\Phi_{S}\right)_{R B}>\left(\Phi_{S}\right)_{R N}>$ $\left(\Phi_{S}\right)_{R U}$ is satisfied. During the calculations of $\left(\Phi_{S}\right)_{R}$ on the basis of Eq. (11), we noticed that the components $R_{11} J_{v}^{2},\left(R_{12}+R_{21}\right) J_{v} J_{s}$ and $R_{22} J_{s}^{2}$ give a different contribution to the global value of $\left(\Phi_{S}\right)_{R}$. It was found that for the same test values $\bar{C}$ the share of individual 


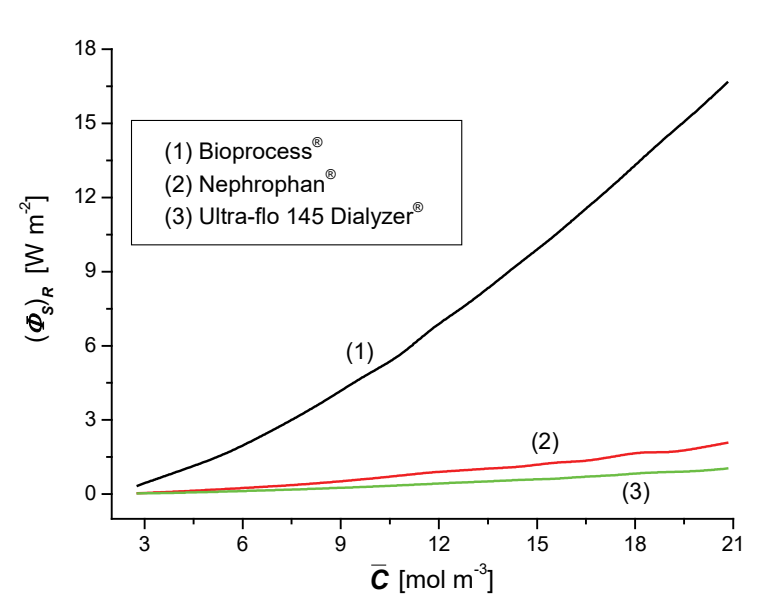

(a)

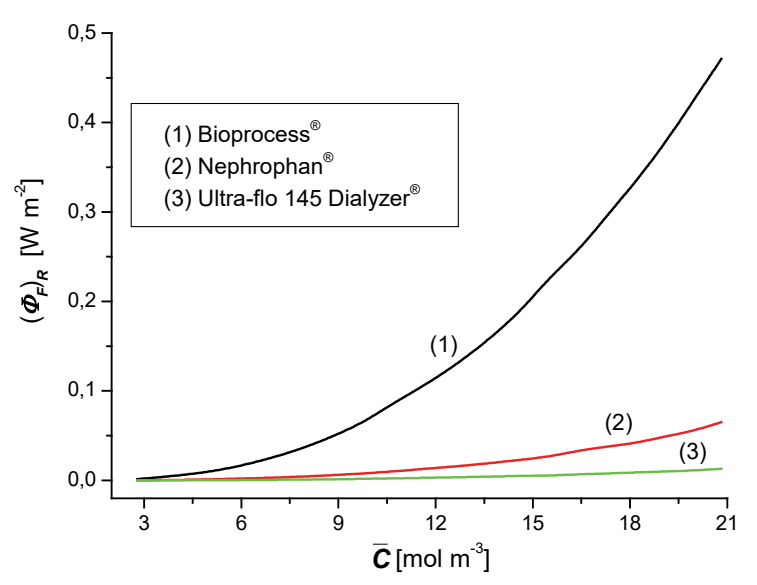

Fig. 7. Graphic illustration of dependencies $\left(\Phi_{S}\right)_{R}=f(\bar{C})(\mathrm{a})$ and $\left(\Phi_{F}\right)_{R}=f(\bar{C})(7 \mathrm{~b})$ for membranes Bioprocess, Nephrophan and Ultra-flo 145 Dialyzer for aqueous glucose solutions

components of Eq. (11) satisfies the relation $R_{22} J_{s}^{2}>$ $\left(R_{12}+R_{21}\right) J_{v} J_{s} \gg R_{11} J_{v}^{2}$.

Based on results of the research presented in Figs. 6 and $7 \mathrm{a}$ and using the Eq. (14), dependencies $\left(\Phi_{F}\right)_{R}=$ $f(\bar{C})$ were calculated. The results of these calculations are shown in Fig. 7b. This figure shows that for the same values of $\bar{C}$, the relation $\left(\Phi_{F}\right)_{R B}>\left(\Phi_{F}\right)_{R N}>$ $\left(\Phi_{F}\right)_{R U}$ is satisfied. Comparing the graphs with the same numbers, it follows that $\left(\Phi_{S}\right)_{R B}>\left(\Phi_{F}\right)_{R B},\left(\Phi_{S}\right)_{R N}>$ $\left(\Phi_{F}\right)_{R N}$ and $\left(\Phi_{S}\right)_{R U}>\left(\Phi_{F}\right)_{R U}$. In systems containing test membranes separating glucose solutions of different concentrations only a small amount of free energy, i.e., useful energy (less than 3\%), can be converted into work (Figs. 7a, b). The rest (97\%) is degraded energy.

\section{Conclusions}

1. The values of the $L_{p}, \omega$ and $\sigma$ coefficients for Bioprocess, Nephrophan and Ultra-flo 145 Dialyzer membranes do not depend on glucose concentrations.

2. The glucose concentration characteristics of the $R_{11}$ Peusner coefficients for the Bioprocess, Nephrophan and Ultra-flo 145 Dialyzer membranes are linear. The glucose concentration characteristics of the $R_{22}$ and $R_{\text {det }}$ coefficients for the same membranes are non-linear. In turn, in the same concentration range of glucose solutions, the coefficients $L_{12}=L_{21}$ for the same membranes are not-depend on glucose concentration $(\bar{C})$.

3. The coefficients of coupling $r_{12}=r_{21}$ as functions of glucose concentration $(\bar{C})$ for Bioprocess, Nephrophan and Ultra-flo 145 Dialyzer membranes have approximately saturation type. The glucose concentration characteristics of the $\left(e_{12}\right)_{r}=\left(e_{21}\right)_{r}$ and $Q_{R}$ Peusner coefficients for this membranes are linear. The highest values of these coefficients were obtained for the Bioprocess and Nephrophan membrane and the lowest - for the Ultra-flo 145 Dialyzer membrane. Moreover, the processes taking place in the system containing this membrane are weakly coupled and there are weak energy conversion interactions.

4. For the tested membranes, the calculated value of free energy production $\left(\Phi_{F}\right)_{R}$ is many times smaller than the value of the energy dissipation function $\left(\Phi_{S}\right)_{R}$ and, e.g., for $\bar{C}=20.8 \mathrm{~mol} \cdot \mathrm{m}^{-3}\left(\Phi_{F}\right)_{R}$ is almost 40 times smaller than $\left(\Phi_{S}\right)_{R}$.

5. The $R$ form of the Kedem-Katchalsky-Peusner model equations, by introducing the Peusner coefficients, enables energy analysis of membrane processes and is a useful tool for studying the transport properties of biomembranes.

\section{References}

[1] BAKER R., Membrane technology and application, John Wiley \& Sons, New York 2012.

[2] BAtKo K.M., ŚlęZAK-ProchazKa I., GrzegorczyN S., ŚLĘZAK A., Membrane transport in concentration polarization conditions: network thermodynamics model equations, J. Porous Med., 2014, 17, 573-586.

[3] BatKo K.M., ŚlęZAK-ProchazKa I., ŚlęZAK A., Network hybrid form of the Kedem-Katchalsky equations for non-homogenous binary non-electrolyte solutions: evaluation of $P_{i j}{ }^{*}$ Peusner's tensor coefficients, Transp. Porous Med., 2015, 106, 1-20.

[4] Batko M., ŚlęzAK A., GrZegorczyn S., Bajdur W.M., The $R^{r}$ form of the Kedem-Katchalsky-Peusner model equations for description of the membrane transport in concentration polarization conditions, Entropy 2020, 22, 857, 1-27. 
[5] CAPLAN S.R., The degree of coupling and its relation to efficiency of energy conversion in multiple-flow systems, J. Theoret. Biol., 1965, 10, 209-235.

[6] Demirel Y., Nonequilibrium thermodynamics: transport and rate processes in physical, chemical and biological systems, Elsevier, Amsterdam 2014.

[7] DzierzKowska E., ŚcisŁowska-CZarnecka A., Matwally S., Romaniszyn D., Chadzińska M., Stodolak-Zych E., Porous poly(lactic acid) based fibres as drug carriers in active dressings, Acta Bioeng. Biomech., 2020, 22, 185-197.

[8] GRZEGORCZYN S., ŚlĘZAK A., Kinetics of concentration boundary layers build up in the system consisted of microbial cellulose biomembrane and electrolyte solutions, J. Membr. Sci., 2007, 304, 148-155.

[9] KATChAlSKY A., CURRAN P.F., Nonequilibrium thermodynamics in biophysics, Harvard University Press, Cambridge 1965.

[10] Kedem O., CaPlan S.R., Degree of coupling and its relation to efficiency of energy conversion, Trans. Faraday Soc., 1965, 61, 1897-1911.

[11] Klinkman H., Holtz M., Willgerodt W., Wilke G., SCHOENFELdER D., Nephrophan - eine neue dialysemembran, Zeit. Urolog., 1969, 4, 285-292.

[12] ONSAGER L., Reciprocal relations in irreversible processes, Phys. Rev., 1931, 78, 405-426.

[13] PeUSNeR L., The principles of network thermodynamics: Theory and biophysical Applications, Harvard University, Ph.D. Thesis, Cambridge, Massachusetts, 1970.

[14] Peusner L., Hierarchies of irreversible energy conversion systems: A network thermodynamics approach. I. Linear steady state without storage, J. Theoret. Biol., 1983, 10, 27-39.

[15] Peusner L., Hierarchies of irreversible energy conversion systems. II. Network derivation of linear transport equations, J. Theoret. Biol., 1985, 115, 319-335.

[16] Peusner L., Studies in network thermodynamics, Elsevier, Amsterdam 1986.
[17] RICHTER T., KEIPERT S., In vitro permeation studies comparing bovine nasal mucosa, porcine cornea and artificial membrane: androstendedione in microemulsions and their components, Europ. J. Pharma. Biopharm., 2004, 58, 137-143.

[18] Twardowski Z., Scholarly Review: History of hemodialyzers' designs, Hemodialysis Inter., 2008, 12, 173-210.

[19] ŚlęzaK A., KucharzewsKi M., FraneK A., TwardokęS W., The evaluation method of effectiveness of healing process of venous leg ulceration, Med. Eng. Phys., 2004, 26, 53-60.

[20] ŚlęZaK-ProchazKa I., BatKo K.M., WąsiK S., ŚlęzaK A., $H^{*}$ Peusner's form of the Kedem-Katchalsky equations fon on-homogeneous non-electrolyte binary solutions, Transp. Porous Med., 2016, 111, 457-477.

[21] ŚlęzAK A., GrZegorCZyn S., BATKo K.M., Resistance coefficients of polymer membrane with concentration polarization, Transp. Porous Med., 2012, 95, 151-170.

[22] ŚlęzAK A., GRZEGorcZyn S., JASIK-ŚLĘZAK J., MichalSKAMAŁECKA K., Natural convection as an asymmtrical factor of the transport through porous membrane, Trans. Porous Med., 2010, 84, 685-698.

[23] ŚlęzaK A., ŚlęzaK-ProchazKa I., GrZegorczyn S., JasiKŚLĘZAK J., Evaluation of S-entropy production in a singlemembrane system in concentration polarization conditions, Trans. Porous Med., 2017, 116, 941-957.

[24] Ślęzak A., GrZegorczyn S., ŚlęzaK I.H., Bryll A., Study of the volume and solute flows through double-membraneous polymeric dressing with silver ions, J. Membr. Sci., 2006, 285, 68-74.

[25] ŚlezaK A., Grzegorczyn S., Batko K., Pilis W., BiczaK R., Membrane transport in concentration polarization conditions: Evaluation of S-entropy production for ternary non-electrolyte solutions, J. Non-Equilib. Thermodyn., 2020, 45, 385-399.

[26] Ullah H., Santos H.A., Khan T., Application of bacterial cellulose in food, cosmetics and drug delivery, Cellulose, 2016, 23, 2291-2314.

[27] WINNE D., Unstirred layer, source of biased Michaelis constant in membrane transport, Biochem. Biophys. Acta, 1973, 298, 27-31. 